\title{
RECENT CO(2-1) OBSERVATIONS OF GALAXIES WITH THE CSO
}

\author{
A. I. SARGENT, T. G. PHILLIPS, D. B. SANDERS, N. Z. SCOVILLE \\ California Institute of Technology \\ Pasadena, CA 91125
}

ABSTRACT $\mathrm{CO}(2 \rightarrow 1)$ observations of a number of galaxies have recently been obtained with the CSO. Examples are presented here to illustrate the capabilities of the instrument.

\section{Introduction}

The newly-constructed Caltech Submillimeter Observatory (CSO) on Mauna Kea, Hawaii, has been used to acquire observations of several galaxies in the $\mathrm{CO}(2 \rightarrow 1)$ transition at $230 \mathrm{GHz}$. Currently, the $10.4 \mathrm{~m}$ diameter Leighton telescope is equipped with an SIS receiver with double-sideband noise temperature $\sim 200 \mathrm{~K}$, and an acousto-optic spectrometer of bandwidth $500 \mathrm{MHz}$. The high surface accuracy dish, $30 \mu \mathrm{m}$, and the excellent atmospheric transmission make it well-suited for sensitive measurements of galaxies. Observations presented here demonstrate the versatility of the system and its potential for studying distant galaxies.

\section{Results}

New $\mathrm{CO}(2 \rightarrow 1)$ observations of Centaurus A (NGC 5128) have been made to compliment the initial 7-point map of Phillips et al. (1987). These confirm that the molecular gas is rotating at velocities of a few hundred $\mathrm{km} \mathrm{s}^{-1}$ in a direction perpendicular to the ellipsoidal stellar component (c.f. Wilkinson et al. 1986), reflecting the separate dynamical systems in this highly disturbed merger. They also allow an examination of the kinematics of the central core, where HI VLA observations (van Gorkom 1987) are handicapped by self-absorption. The molecular gas and far infrared emission distributions (Joy et al. 1988) are remarkably similar.

NGC 3256, whose characteristic "tails" and chaotic nuclear appearance at optical wavelengths clearly identify it as a merging system (Toomre 1977; Schweizer 1986), has also been studied. Molecular gas extends over $7-10 \mathrm{kpc}$ around the nucleus, as does $10 \mu \mathrm{m}$ emission (Graham et al. 1987), indicating that the enhanced luminosity seen by IRAS, $3.2 \times 10^{11} \mathrm{~L}_{\odot}$, is plausibly accounted for by a burst of star formation. Although the total mass of gas, $2 \times 10^{10} \mathrm{M}_{\odot}$, is a factor of two higher than that associated with Arp 220, the order of magnitude lower luminosity suggests that in NGC 3256 the merger is less advanced (c.f. Joseph and Wright 1985). Indeed, ESO photographic plates, kindly provided by J. Bergeron, show two central condensations separated by only $4^{\prime \prime}$, which could reflect the presence of independent nuclei.

$\mathrm{CO}(2 \rightarrow 1)$ observations of four more distant galaxies, NGC $1614\left(\mathrm{cz} \approx 4800 \mathrm{~km} \mathrm{~s}^{-1}\right)$, NGC $2623\left(\mathrm{cz} \approx 5500 \mathrm{~km} \mathrm{~s}^{-1}\right)$, NGC $6090\left(\mathrm{cz} \approx 6090 \mathrm{~km} \mathrm{~s}^{-1}\right)$, and Markarian $231(\mathrm{cz} \approx 12700 \mathrm{~km}$

D. McNally (ed.), Highlights of Astronomy, Vol. 8, 591-592.

(C) 1989 by the IAU. 
$\mathrm{s}^{-1}$ ), have also been made. For the nearer objects, typical integration times were about 25 minutes, while the spectrum of Mrk 231 shown in Figure 1 was acquired in an hour.

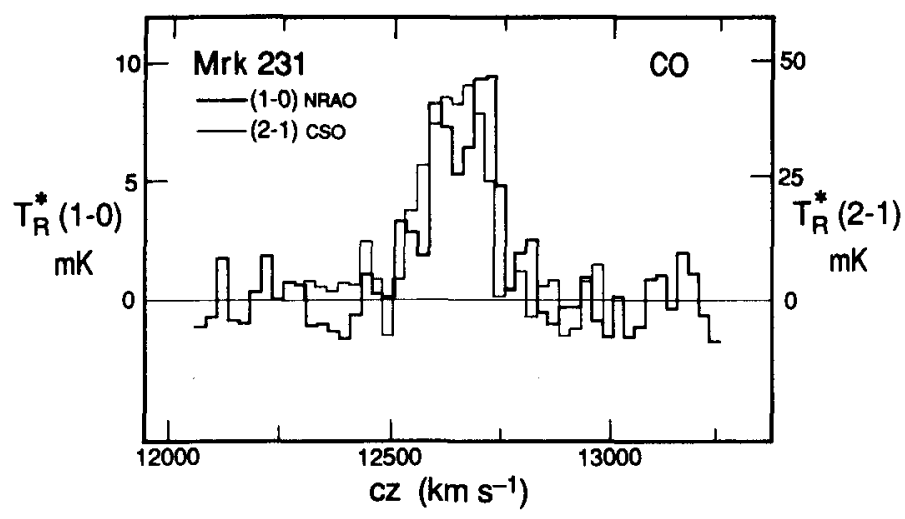

Figure 1. A spectrum of the $\mathrm{CO}(2 \rightarrow 1)$ emission from Markarian 231 acquired at the CSO, superimposed on the $\mathrm{CO}(1 \rightarrow 0)$ spectrum from $N R A O$

These CSO $\operatorname{CO}(2 \rightarrow 1)$ spectra have been compared with $\operatorname{CO}(1 \rightarrow 0)$ profiles obtained with the NRAO 12-meter telescope. The results for Mrk 231, displayed in Figure 1, are typical of galaxies unresolved by both instruments; the line shapes are very similar and the substantially higher values of $T_{R}^{*}$ for the $\operatorname{CO}(2 \rightarrow 1)$ line probably reflect only the ratio of telescope beam size. Thus we have as yet no evidence that the molecular gas, even in these distant galaxies, differs in its properties from molecular clouds in our Milky Way.

\section{References}

van Gorkom, J. 1987, in I. A. U. Symposium No. 127, Structure and Dynamics of Elliptical Galaxies, ed. T. de Zeeuw (Dordrecht:Reidel), p. 421.

Graham, J. R., Wright, G. S., Joseph, R. D., Frogel, J. A., Phillips, M. M., and Meikle, W. P. S. 1987, in Star Formation in Galaxies, ed. C. J. Lonsdale (U. S. Government Printing Office), p. 517.

Joseph, R. D., and Wright, G. S. 1985, M. N. R. A. S., 214, 87.

Joy, M., Lester, D. F., Harvey, P. M., and Ellis, H. B. 1988, Ap. J., 326, 662.

Phillips, T. G. et al. 1987, 322, L73.

Schweizer, F. 1986, Science, 231, 227.

Toomre, A. 1977, in The Evolution of Galaxies and Stellar Populations, (Yale University Observatory, New Haven), p. 401.

Wilkinson, A., Sharples, R. M., Fosbury, R. A. E., and Wallace, P. T. 1986, M. N. R. A. S., 218, 297. 